\title{
On the asymptotic power of a goodness-of-fit test based on a cumulative Kullback-Leibler discrepancy
}

\author{
Contreras-Cristán, A. ${ }^{\mathrm{a}, *}$, Gutiérrez-Peña, E. ${ }^{\mathrm{a}}$, Walker, S.G. ${ }^{\mathrm{b}}$ \\ ${ }^{a}$ Department of Probability and Statistics, IIMAS-UNAM, Mexico. \\ ${ }^{b}$ Department of Mathematics $\&$ Department of Statistics and Data Science \\ University of Texas at Austin, USA.
}

\begin{abstract}
We discuss a goodness-of-fit test arising from information-theoretical considerations. We show that, in the simple case, our test has superior asymptotic power compared to the Anderson-Darling test when the alternative lies in a certain large class of distribution functions.
\end{abstract}

Keywords: EDF statistic, Information measure, Power of a test.

${ }^{*}$ Corresponding author: Alberto Contreras-Cristán, Departamento de Probabilidad y Estadística, IIMAS-UNAM, Apdo. Postal 20-126, México D.F. 01000, Mexico.

Tel. +52 55 5622-3485. E-mail: alberto@sigma.iimas.unam.mx.

Preprint submitted to Elsevier

September 26, 2016

(C) 2016. This manuscript version is made available under the Elsevier user license http://www.elsevier.com/open-access/userlicense/1.0/ 


\section{Introduction}

A number of goodness-of-fit tests based on the comparison of $F_{0}$ (the distribution function assumed under the null hypothesis) with the empirical distribution function have been proposed. Some of the EDF (Empirical Distribution Function) statistics obtained correspond to well-known tests such as Kolmogorov-Smirnoff, Cramer-von Mises and Anderson-Darling. Of these, the Anderson-Darling test (Anderson and Darling, 1954) is regarded as one of the most powerful to test $F_{0}$ against a wide range of alternatives (see, for example, D'Agostino and Stephens, 1986).

In this paper, we propose a test statistic derived from a cumulative discrepancy based on the Kullback-Leibler divergence between $F_{0}$ and a Bayesian-motivated version of the empirical distribution function. The latter was proposed by Hill (1968) as a posterior predictive distribution for exchangeable observations under a nonparametric prior distribution for the unknown distribution $F$.

Our test improves uniformly on the power function, compared to the Anderson-Darling test, for a class of tests involving the null hypothesis $H_{0}: F=F_{0}$ (with $F_{0}$ fully specified) when the true distribution under the alternative hypothesis belongs to a large class of distribution. This class is given by

$$
\mathcal{S}=\left\{F: \int_{0}^{1} \frac{G(z)(1-G(z))}{z(1-z)} \mathrm{d} z \geq 1\right\},
$$

where

$$
G(z)=F\left(F_{0}^{-1}(z)\right) \quad(0<z<1) .
$$

One of the main aims of this paper is to study the form of the distributions in $\mathcal{S}$.

The layout of the paper is as follows. In Section 2 we introduce the new test. Section 3 then provides details of how it is similar to the AndersonDarling test; yet has crucial differences. In Section 4 we prove the superiority of our test for alternatives in the class $\mathcal{S}$, and briefly discuss the asymptotic relative efficiency of both tests. We then investigate $\mathcal{S}$ in Section 5. Finally, Section 6 contains some simulation studies.

\section{The test}

Let us assume that, for a random sample $\left(x_{1}, \ldots, x_{n}\right)$ of size $n$, the true distribution function is $F$ and we are interested in testing the simple hypoth- 
esis $H_{0}: F=F_{0}$, where $F_{0}$ is a fully-specified continuous distribution. As usual in the simple case, we will use $z_{(i)}=F_{0}\left(x_{(i)}\right)$, for $i=1, \ldots, n$, where $\left(x_{(i)}: i=1, \ldots, n\right)$ denote the order statistics.

Given these, consider the intervals $I_{0}=\left(-\infty, x_{(1)}\right), I_{i}=\left(x_{(i)}, x_{(i+1)}\right)$ for $i=1, \ldots, n-1$, and $I_{n}=\left(x_{(n)}, \infty\right)$. Hill (1968) suggested a nonparametric posterior predictive model which assigns mass $1 /(n+1)$ to the event that a new observation will fall within $I_{i}$. Under this model, the probability assigned to the interval $\left(0, z_{(i)}\right)$ is $p_{i}=i /(n+1)$. On the other hand, under the null hypothesis, the probability assigned to $\left(0, z_{(i)}\right)$ is $z_{(i)}$.

For each $i$, a measure of discrepancy between the Bernoulli probability distributions $\left\{p_{i}, 1-p_{i}\right\}$ and $\left\{z_{(i)}, 1-z_{(i)}\right\}$ is given by the Kullback-Leibler divergence $D\left(p_{i}, z_{(i)}\right)=p_{i} \log \left(p_{i} / z_{(i)}\right)+\left(1-p_{i}\right) \log \left(\left(1-p_{i}\right) /\left(1-z_{(i)}\right)\right)$. We add these discrepancies to obtain the cumulative test statistic

$$
K=\sum_{i=1}^{n} D\left(p_{i}, z_{(i)}\right)=\sum_{i=1}^{n}\left\{p_{i} \log \left(p_{i} / z_{(i)}\right)+\left(1-p_{i}\right) \log \left(\left(1-p_{i}\right) /\left(1-z_{(i)}\right)\right)\right\} .
$$

\section{The relationship between $K$ and the Anderson-Darling test}

To explore the connection with the Anderson-Darling statistic, it is convenient to write $K$ as

$$
K=A_{n}-(n+1)^{-1} \sum_{i=1}^{n} i\left[\log z_{(i)}+\log \left(1-z_{(n+1-i)}\right)\right]
$$

where $A_{n}=\sum_{i=1}^{n}\left[p_{i} \log p_{i}+\left(1-p_{i}\right) \log \left(1-p_{i}\right)\right]$. The Anderson-Darling statistic can be written as

$$
A^{2}=-n-n^{-1} \sum_{i=1}^{n}\left[(2 i-1)\left(\log z_{(i)}+\log \left(1-z_{(n+1-i)}\right)\right)\right]
$$

see D'Agostino and Stephens (1986; p. 101). On the other hand, note that

$$
A^{2}=-n-B_{n}+2 \sum_{i=1}^{n} D\left(p_{i}^{*}, z_{(i)}\right)
$$

where $p_{i}^{*}=(2 i-1) /(2 n)$ and $B_{n}=2 \sum_{i=1}^{n}\left[p_{i}^{*} \log p_{i}^{*}+\left(1-p_{i} *\right) \log \left(1-p_{i}^{*}\right)\right]$. Since the properties of the tests do not depend on constants such as $n$ and $B_{n}$, without loss of generality we can work with the Anderson-Darling test 
in terms of the statistic $\mathcal{A}^{2}=\sum_{i=1}^{n} D\left(p_{i}^{*}, z_{(i)}\right)$. The statistic $K$ uses $p_{i}=$ $i /(n+1)$, while $\mathcal{A}^{2}$ uses $p_{i}^{*}=(2 i-1) /(2 n)$. The $p_{i}$ 's have equal spacings of $1 /(n+1)$ which is the form of the $z_{(i)}$ under a "perfect sample". The $p_{i}^{*}$ 's on the other hand have equal spacings of $1 / n$, save for the first and last intervals, which are of size $1 /(2 n)$.

Before developing the asymptotic theory, we note that $\left(B_{n}+n\right) / n \rightarrow 0$ as $n \rightarrow \infty$. To see this, first write

$$
\frac{B_{n}}{n}=\frac{2}{n}\left\{2 \sum_{i=1}^{n} p_{i}^{*} \log \left(p_{i}^{*}\right)\right\}=\frac{4}{n} \sum_{i=1}^{n}\left(\frac{i}{n}-\frac{1}{2 n}\right) \log \left(\frac{i}{n}-\frac{1}{2 n}\right) .
$$

The right hand side of the equation above is a Riemann sum approximating the integral $4 \int_{0}^{1} y \log (y) d y,=-1$, and therefore $\lim _{n \rightarrow \infty} B_{n} / n=-1$. From this result and equation (3), we get that, with probability 1 ,

$$
\frac{A^{2}}{n}-\frac{2 \mathcal{A}^{2}}{n} \rightarrow 0, \quad \text { as } n \rightarrow \infty .
$$

\section{Asymptotic comparison of the tests}

We will consider the power of $K$ and $\mathcal{A}^{2}$ for alternatives $F \in \mathcal{S}$, where $\mathcal{S}$ is defined by (1). Let $g(z)$ be the density function corresponding to the c.d.f. $G(z)$ defined in (2). We then have the following.

Lemma 1. Let $D_{n}=K-\mathcal{A}^{2}=\sum_{i=1}^{n}\left[D\left(p_{i}, z_{(i)}\right)-D\left(p_{i}^{*}, z_{(i)}\right)\right]$. If

$$
\int_{0}^{1}\left|\log \left(\frac{z}{1-z}\right)\right|^{2} g(z) \mathrm{d} z<\infty
$$

then, as $n \rightarrow \infty, D_{n} \rightarrow D(G)-\frac{1}{2} \quad$ (a.s.) where

$$
D(G)=\frac{1}{2} \int_{0}^{1} \frac{G(z)(1-G(z))}{z(1-z)} \mathrm{d} z .
$$

Proof. First note that $D_{n}=C_{n}+\sum_{i=1}^{n}\left(p_{i}^{*}-p_{i}\right) \log \left\{z_{(i)} /\left(1-z_{(i)}\right)\right\}$, where

$C_{n}=\sum_{i=1}^{n} p_{i} \log p_{i}+\sum_{i=1}^{n}\left(1-p_{i}\right) \log \left(1-p_{i}\right)-\sum_{i=1}^{n} p_{i}^{*} \log p_{i}^{*}-\sum_{i=1}^{n}\left(1-p_{i}^{*}\right) \log \left(1-p_{i}^{*}\right)$ 
does not depend on the $\left\{z_{(i)}\right\}$, and $p_{i}^{*}-p_{i}=[i /(n(n+1))]-[1 /(2 n)]$. Let us write

$D_{n}^{\prime}=\frac{1}{n(n+1)} \sum_{i=1}^{n} i \log \left(z_{(i)} /\left(1-z_{(i)}\right)\right)-\frac{1}{2 n} \sum_{i=1}^{n} \log \left(z_{(i)} /\left(1-z_{(i)}\right)\right):=D_{1 n}-D_{2 n}$.

For the second of these two terms, by the Law of Large Numbers we have

$$
D_{2 n} \rightarrow \frac{1}{2} \int_{0}^{1} \log (z /(1-z)) g(z) \mathrm{d} z \quad \text { (a.s.) }
$$

For the first term, we use the fact that the density function for $z_{(i)}$ is given by

$$
f_{z_{(i)}}(z)=\frac{n !}{(i-1) !(n-i) !} G(z)^{i-1}(1-G(z))^{n-i} g(z)
$$

Now,

$$
\left|D_{1 n}\right| \leq n^{-1} \sum_{i=1}^{n}\left|\log \left(\frac{z_{(i)}}{1-z_{(i)}}\right)\right|
$$

and the right-hand side has the expected value

$$
\int_{0}^{1}\left|\log \left(\frac{z}{1-z}\right)\right| g(z) \mathrm{d} z
$$

Thus, if this is finite then $D_{1 n}$ converges almost surely. Specifically, we have

$$
n^{-1} \sum_{i=1}^{n} \frac{i n !}{(i-1) !(n-i) !} G(z)^{i-1}(1-G(z))^{n-i}=1+(n-1) G(z)
$$

and hence

$$
\mathrm{E}\left(D_{1 n}\right)=\frac{1}{n+1} \int_{0}^{1}\{1+(n-1) G(z)\} g(z) \log (z /(1-z)) \mathrm{d} z .
$$

This converges, and a trivial calculation gives $\operatorname{Var}\left(D_{1 n}\right) \rightarrow 0$, so

$$
D_{1 n} \rightarrow \int_{0}^{1} \log (z /(1-z)) G(z) g(z) \mathrm{d} z \quad \text { (a.s.) }
$$

Putting the two limits (5) and (6) together, we have

$$
D_{n}^{\prime} \rightarrow \frac{1}{2} \int_{0}^{1} \log (z /(1-z)) g(z)\{2 G(z)-1\} \mathrm{d} z \quad \text { (a.s.) }
$$


Using integration by parts, the limit (7) can be written as $D(G)$.

Finally, we show that $C_{n} \rightarrow-\frac{1}{2}$ as $n \rightarrow \infty$. Note that $C_{n}$ can be written as

$2(n+1)\left\{\frac{1}{n+1} \sum_{i=1}^{n}\left(\frac{i}{n+1}\right) \log \left(\frac{i}{n+1}\right)\right\}-2 n\left\{\frac{1}{n} \sum_{i=1}^{n}\left(\frac{i}{n}-\frac{1}{2 n}\right) \log \left(\frac{i}{n}-\frac{1}{2 n}\right)\right\}$.

Each of the terms in brackets is a Riemann sum approximating the integral $\int_{0}^{1} y \log (y) \mathrm{d} y=-1 / 4$. Thus, for large $n$, we have $C_{n} \approx 2(n+1)\left\{-\frac{1}{4}\right\}-$ $2 n\left\{-\frac{1}{4}\right\}=-\frac{1}{2}$; see Theorem 1-c in Chui (1971). This concludes the proof. $\diamond$

Without loss of generality, we can compare the $K$ and $\mathcal{A}^{2}$ tests in the case where $Z=F_{0}(X)$ is uniform $\mathrm{U}(0,1)$ under the null hypothesis and has distribution $G(z)$ under the alternative. Note that $D(\mathrm{U}(0,1))=\frac{1}{2}$.

Both $K$ and $\mathcal{A}^{2}$ diverge as $n \rightarrow \infty$ under any alternative. However, it can be shown that both $n^{-1} K(G)$ and $n^{-1} \mathcal{A}^{2}(G)$ converge; see expressions (8) and (11) below. Thus,

$$
n^{-1} K(G)=n^{-1} \mathcal{A}^{2}(G)+n^{-1}\left\{D(G)-\frac{1}{2}\right\}+o\left(n^{-1}\right)
$$

and so if $D(G)>\frac{1}{2}$ then $K$ improves on the power of $\mathcal{A}^{2}$ to a first order approximation.

We now turn our attention to the asymptotic efficiency of the tests. Specifically, we study the asymptotic relative efficiency using the approach of Bahadur (1967). Suppose $F$ is a continuous distribution in a class $\mathcal{F}_{\Theta}=$ $\left\{F_{\theta}: \theta \in \Theta\right\}$ indexed by a parameter $\theta$ such that $H_{0}: F_{\theta}=F_{0}$.

Given a value of $\theta$ in $\Theta_{1}=\Theta-\left\{\theta_{0}\right\}$,

$$
\frac{A^{2}}{n} \stackrel{p}{\longrightarrow} b(\theta)=\int_{-\infty}^{\infty} \frac{\left(F_{\theta}-F_{0}\right)^{2}}{F_{0}\left(1-F_{0}\right)} d F_{0}
$$

as $n \rightarrow \infty$; see, for example, Grané and Tchirina (2013). It then follows from (3) that, as $n \rightarrow \infty$,

$$
\frac{2 \mathcal{A}^{2}}{n} \stackrel{p}{\longrightarrow} b(\theta)
$$

Using Theorem 1.2.2 of Nikitin (1995), the main term of the exact Bahadur slope corresponding to the Anderson-Darling statistic $A^{2}$ is given by

$$
c_{A^{2}}(\theta)=2 \int_{-\infty}^{\infty} \frac{\left(F_{\theta}-F_{0}\right)^{2}}{F_{0}\left(1-F_{0}\right)} d F_{0},
$$


for $\theta \in \Theta_{1}$; see Grané and Tchirina (2013). Note that from (4) we have

$$
\lim _{n \rightarrow \infty} \frac{1}{n} \log P\left(\frac{2 \mathcal{A}^{2}}{n}>t\right)=\lim _{n \rightarrow \infty} \frac{1}{n} \log P\left(\frac{A^{2}}{n}>t\right)=-t+o(t),
$$

where, for sufficiently small $t>0$, the last equality follows from Nikitin (1995; Theorem 2.5.2). Expressions (8) and (9) then allow us to use Theorem 1.2.2 of Nikitin (1995) to show that $A^{2}$ and $2 \mathcal{A}^{2}$ share the same main term of their exact Bahadur slopes, i.e. $c_{2 \mathcal{A}^{2}}(\theta)=c_{A^{2}}(\theta)$. An analogous argument leads to the conclusion that $c_{\mathcal{A}^{2}}(\theta)=c_{A^{2}}(\theta)$. Now, assuming the conditions ensuring Lemma 1 hold, we have

$$
\frac{K}{n}-\frac{\mathcal{A}^{2}}{n} \rightarrow 0 \quad \text { (a.s.) }
$$

as $n \rightarrow \infty$. This, together with expression (8), imply that

$$
\frac{K}{n} \stackrel{p}{\longrightarrow} \frac{1}{2} b(\theta)
$$

as $n \rightarrow \infty$. Also, from (10), for sufficiently small $u>0$,

$$
\lim _{n \rightarrow \infty} \frac{1}{n} \log P\left(\frac{K}{n}>u\right)=\lim _{n \rightarrow \infty} \frac{1}{n} \log P\left(\frac{\mathcal{A}^{2}}{n}>u\right)=2 u+o(2 u) .
$$

Again, from Theorem 1.2.2 of Nikitin (1995) we conclude that $A^{2}$ and $K$ have the same main term of their exact Bahadur slopes, so that $c_{A^{2}}(\theta)=c_{K}(\theta)$. Thus, $A^{2}$ and $K$ have the same local Bahadur Efficiency.

\section{The class $\mathcal{S}$}

In this section we aim to characterize cases where $D(G) \geq \frac{1}{2}$. We consider two broad situations:

1. Overdispersion: i.e., $F_{0}$ and $F$ have the same median (at 0 , without loss of generality) and $F(x) \geq F_{0}(x)$ for $x \leq 0$ while $F(x) \leq F_{0}(x)$ for $x \geq 0$.

2. Heavy tails: $F(x)=F_{0}(x)^{c}$ for $0<c<1$, which implies $F$ has heavier tails (notably for small values of $F_{0}$, i.e. the left tail). Similarly, $F(x)=$ $1-\left(1-F_{0}(x)\right)^{c}$ implies $F$ has heavier tails on the right. Finally, $F$ a mixture of these two will imply $F$ has heavier tails compared to $F_{0}$. 
Theorem 1. If $F_{0}$ and $F$ have median at $0, F(x) \geq F_{0}(x)$ for all $x \leq 0$ and $F(x) \leq F_{0}(x)$ for all $x \geq 0$, then $D(G) \geq \frac{1}{2}$.

Proof. Recall that $G(z)=F\left(F_{0}^{-1}(z)\right)$. Since $\frac{1}{2} \geq G(z) \geq z$ for $0 \leq z \leq \frac{1}{2}$, $\frac{1}{2} \leq G(z) \leq z$ for $\frac{1}{2} \leq z \leq 1$, and $G\left(\frac{1}{2}\right)=\frac{1}{2}$, it is easy to show that

$$
G(z)(1-G(z)) \geq z(1-z) \text { for all } 0 \leq z \leq 1 .
$$

Hence $D(G) \geq \frac{1}{2}$.

A special instance of this scenario arises when we compare variances, specifically when testing against a larger variance. In this case we have $F(x)=F_{0}(x / \sigma)$ with $\sigma>1$. Then $x \geq 0$ implies $x / \sigma \leq x$ which implies $F_{0}(x / \sigma) \leq F_{0}(x)$, while $x \leq 0$ implies $x / \sigma \geq x$ which in turn implies $F_{0}(x / \sigma) \geq F_{0}(x)$.

We now turn our attention to the case where $F$ has heavy tails compared to $F_{0}$. In this case $G(z)$ has a bathtub-shaped density function $g(z)$ on $(0,1)$. We can construct such densities using mixtures of $\operatorname{beta}(c, 1)$ and $\operatorname{beta}(1, c)$ densites with $0<c<1$. To achieve this we need to prove two results: The first is that if $G(z)=z^{c}$ or $G(z)=1-(1-z)^{c}$, with $0<c<1$, then $D(G) \geq \frac{1}{2}$; and the second is that if $G^{*}$ and $G^{\dagger}$ satisfy $D\left(G^{*}\right)>\frac{1}{2}$ and $D\left(G^{\dagger}\right)>\frac{1}{2}$, then for any $0<w<1$ it holds that $D\left(w G^{*}+(1-w) G^{\dagger}\right)>\frac{1}{2}$. Note that

$$
\int_{0}^{1}\left|\log \left(\frac{z}{1-z}\right)\right|^{2} z^{c-1} \mathrm{~d} z<\infty
$$

for all $0<c<1$ so, by Lemma $1, D_{n} \rightarrow D(G)-\frac{1}{2}$.

Theorem 2. If $G(z)=z^{c}$ for some $0<c<1$ then $D(G) \geq \frac{1}{2}$. Moreover, if $D\left(G^{*}\right) \geq \frac{1}{2}$ and $D\left(G^{\dagger}\right) \geq \frac{1}{2}$ then, for any $w$ such that $0<w<1$, we have $D\left(w G^{*}+(1-w) G^{\dagger}\right)>\frac{1}{2}$.

Proof. For the first part, note that

$$
T(c) \equiv \int_{0}^{1} \frac{G(z)(1-G(z))}{z(1-z)} \mathrm{d} z=\int_{0}^{1}\left(\frac{z^{c}}{z}\right)\left(\frac{1-z^{c}}{1-z}\right) \mathrm{d} z .
$$

Now, the function $u(z)=\frac{1-z^{c}}{1-z}$ is decreasing in $z$, is such that $u(0)=1$ and (by L'Hospital's rule) $u(z) \rightarrow c$ as $z \rightarrow 1$. Therefore $u(z) \geq c$ for all $z \in(0,1)$ and consequently, for all $c$ in $(0,1)$,

$$
T(c)=\int_{0}^{1}\left(\frac{z^{c}}{z}\right) u(z) \mathrm{d} z \geq c \int_{0}^{1} z^{c-1} \mathrm{~d} z=c\left\{\frac{1}{c}\right\}=1 .
$$


Thus $T(c) \geq 1$ for $0<c \leq 1$, which implies $D(G) \geq \frac{1}{2}$. The same idea applies to showing that $D\left(1-(1-z)^{c}\right) \geq \frac{1}{2}$.

Now let $w \in(0,1)$ and consider

$$
\left[w G^{*}+(1-w) G^{\dagger}\right]\left[1-w G^{*}-(1-w) G^{\dagger}\right]
$$

which is given by

$$
w G^{*}\left(1-w G^{*}\right)+(1-w) G^{\dagger}\left(1-(1-w) G^{\dagger}\right)-2 w(1-w) G^{*} G^{\dagger} .
$$

This in turn can be written as

$$
w G^{*}\left(1-G^{*}\right)+(1-w) G^{\dagger}\left(1-G^{\dagger}\right)+w(1-w)\left(G^{*}-G^{\dagger}\right)^{2}
$$

implying that $D\left(w G^{*}+(1-w) G^{\dagger}\right) \geq \frac{1}{2}$.

It follows from the above that

$$
G(z)=w \sum_{j=1}^{\infty} w_{1 j} z^{c_{1 j}}+(1-w) \sum_{j=1}^{\infty} w_{2 j}\left[1-(1-z)^{c_{2 j}}\right]
$$

where $\left\{w_{1 j}\right\}$ and $\left\{w_{2 j}\right\}$ are such that $\sum_{j=1}^{n} w_{1 j}=\sum_{j=1}^{n} w_{2 j}=1$, is in the class $\mathcal{S}$. For $G$ to be centred at $\frac{1}{2}$; i.e. for $F$ having the same median as $F_{0}$, we have

$$
G(z)=\sum_{j=1}^{\infty} \frac{1}{2} w_{j}\left[z^{c_{j}}+1-(1-z)^{c_{j}}\right] .
$$

Remark. When $D(G)<\frac{1}{2}$, the power of $\mathcal{A}^{2}$ is asymptotically superior to that of $K$. However, of the following four cases, it is only the last case (iv) for which $D(G)<\frac{1}{2}$ : (i) $G$ is concave (e.g. $g(z) \propto z^{c-1}$ for $0<c<1$ ); (ii) $G$ is convex (e.g. $g(z) \propto(1-z)^{c-1}$ for $0<c<1$ ); (iii) $G$ is concave then convex (e.g. $g(z) \propto[z(1-z)]^{-1 / 2}$ ); and (iv) $G$ is convex then concave (e.g. $g(z) \propto z(1-z))$; see Figure 1. Cases (i) to (iii) arise when $F$ is overdispersed, has larger variance or heavier tails with respect to $F_{0}$, which are the more usual alternatives of interest.

We close this section by presenting another general class for which $D(G)>$ $\frac{1}{2}$. Let $H(z)$ be a function on $(0,1)$ for which

$$
\int_{0}^{1} H(z) \mathrm{d} z>1 \quad \text { and } \quad z(1-z) H(z) \leq 1 / 4 .
$$


(i)

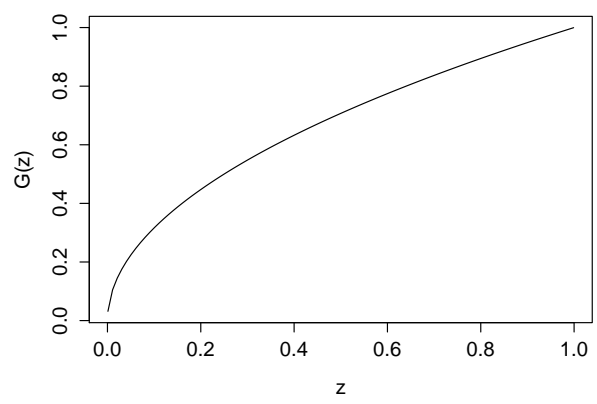

(iii)

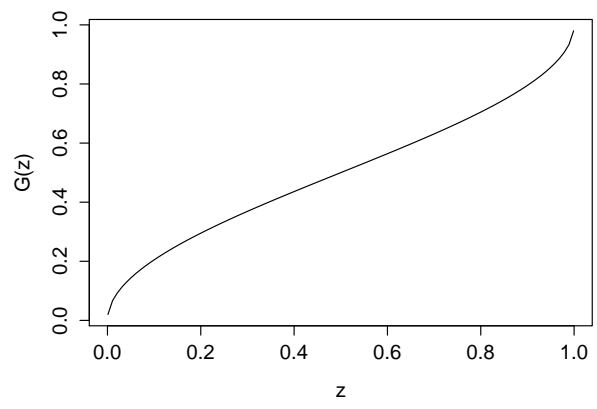

(ii)

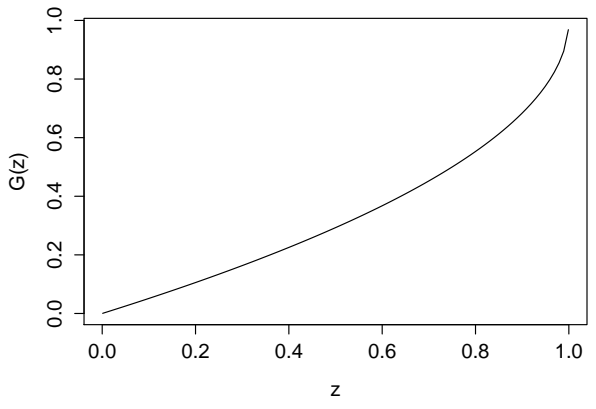

(iv)

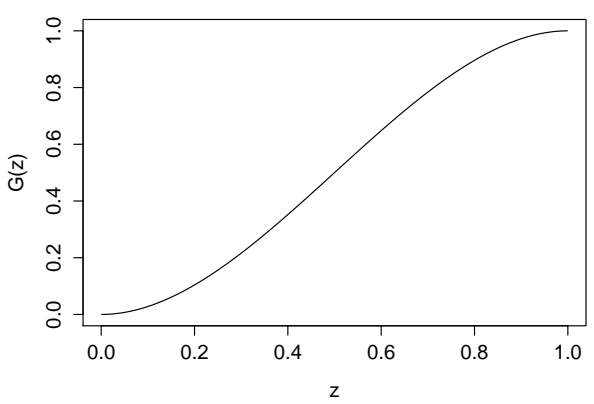

Figure 1: Shapes of $G(z)=F\left(F_{0}^{-1}(z)\right)$.

Then, provided $z(1-z) H(z)$ is increasing for $z \leq \frac{1}{2}$ and decreasing for $z>\frac{1}{2}$,

$$
G(z)=G_{H}(z) \equiv \begin{cases}\frac{1}{2}-\sqrt{1 / 4-z(1-z) H(z)} & z \leq \frac{1}{2} \\ \frac{1}{2}+\sqrt{1 / 4-z(1-z) H(z)} & z>\frac{1}{2}\end{cases}
$$

is a distribution function on $(0,1)$ which has $D(G)>\frac{1}{2}$. To see this, we simply note that $G(z)(1-G(z))=z(1-z) H(z)$. One example is $H(z)=1+\epsilon\left(z-\frac{1}{2}\right)^{2}$ for suitably small $\epsilon$ ensuring $z(1-z) H(z) \leq 1 / 4$.

Another example involves $H(z)=a z^{b-1}(1-z)^{c-1}$ where, in order to satisfy the constraints required, we need

$$
a \frac{\Gamma(b) \Gamma(c)}{\Gamma(b+c)}>1 \quad \text { and } \quad a\left(\frac{b}{b+c}\right)^{b}\left(\frac{c}{b+c}\right)^{c}<1 / 4
$$

Note that $G(z)$ is increasing for $z<b /(b+c)$ and decreasing for $z>b /(b+c)$. If $b=c$ then we need $a<4^{b-1}$ and $0<b<1$; in this case $G(z)$ resembles case (iii) listed above. 


\section{Simulation studies}

Although the theoretical power calculations described in this paper are asymptotic, the improvement in power for the cases we have covered appears to hold also for finite $n$. To show this, here we present a small simulation study involving testing for a uniform $\mathrm{U}(0,1)$ distribution against: (a) mixture alternatives of the form $G(z)=\frac{1}{2} G^{*}(z)+\frac{1}{2} G^{\dagger}(z)$, as discussed in Theorem 2; and (b) the class of alternatives discussed at the end of the previous section. A further simulation study is provided, where we assume a null hypothesis of normality and consider a number of symmetric alternative distributions which are overdispersed relative to the null (see Theorem 1).

Consider the test

$$
\begin{aligned}
& H_{0}: \text { The sample comes from a } \mathrm{U}(0,1) \text { distribution } \\
& \text { vs. } \\
& H_{1}: \quad \text { The sample comes from } G(z) .
\end{aligned}
$$

For case (a), the alternative distribution is given by the mixture of beta densities

$$
g(z)=\frac{1}{2} \operatorname{beta}(z ; c, 1)+\frac{1}{2} \operatorname{beta}(z ; 1, c),
$$

where $\operatorname{beta}(z ; a, b)$ denotes the density function of a beta distribution with parameters $a$ and $b$. Note that this corresponds to using $G^{*}(z)=z^{c}, G^{\dagger}(z)=$ $1-(1-z)^{c}$ and $w=\frac{1}{2}$ in Theorem 2. The left panel of Table 1 shows the power comparison between $K$ and $A^{2}$ when $c=0.7$, for different sample sizes $(n=5,10,20,30,50,90)$. The Kullback-Leibler test turns out to be more powerful than the Anderson-Darling test for all the sample sizes considered.

\begin{tabular}{lccc|cccc}
\hline $\begin{array}{c}\text { Alternative } \\
\text { distribution }\end{array}$ & $n$ & $K$ & $A^{2}$ & $\begin{array}{c}\text { Alternative } \\
\text { distribution }\end{array}$ & $n$ & $K$ & $A^{2}$ \\
\hline (a) Mixture & 5 & 21 & 17 & (b) $G_{H}(z)$ & 5 & 24 & 21 \\
& 10 & 23 & 18 & & 10 & 30 & 25 \\
& 20 & 25 & 20 & & 20 & 40 & 33 \\
& 30 & 28 & 22 & & 30 & 49 & 42 \\
& 50 & 34 & 27 & & 50 & 66 & 60 \\
& 90 & 44 & 37 & & 90 & 88 & 84 \\
\hline
\end{tabular}

Table 1: Power comparisons for test statistics $K$ and $A^{2}$. 
As for case (b), we consider $G(z) \equiv G_{H}(z)$ defined by taking $H(z)=$ $1+3\left(z-\frac{1}{2}\right)^{2}$. The right panel of Table 1 shows the corresponding power comparison. As in the previous case, $K$ proves to be more powerful than $A^{2}$ for all sample sizes under consideration.

These results were obtained by simulating samples of size $n$ from $G$; then, for each of these samples, we computed both test statistics $K$ and $A^{2}$ and performed the corresponding tests. In order to obtain the quantiles of the sampling distribution of the test statistics under $H_{0}$, we simulated $m=100,000$ samples. Similarly, the power of the tests was computed using $k=100,000$ additional simulated samples. The figures reported in Table 1 are the percentage of tests for which $H_{0}$ was rejected at the significance level $\alpha=0.10$ for each sample size $n$.

We now proceed to show the results of another simulation study comparing the Kullback-Leibler and the Anderson-Darling tests for a number of overdispersed and heavy-tailed alternatives. Specifically, we consider the situation where the variance of the alternative distribution is misspecified and greater than the variance assumed under the null hypothesis.

We tested the null hypothesis

$$
H_{0} \text { : The sample comes from a } \operatorname{Normal}(0,2) \text { distribution, }
$$

and considered different alternatives $F$ and sample sizes $n$, as shown in Table 2. We simulated samples of size $n=5,30,50,90$ from $F$; then, for each sample, we computed both $K$ and $A^{2}$. The figures in Table 2 represent the percentage of tests for which $H_{0}$ was rejected at the significance level $\alpha=0.10$. All the alternatives considered have mean zero and a variance of 3 , which is larger than the variance under the null hypothesis. The alternative labelled "Mixture", corresponds to the mixture of three Normal distributions $f(x)=0.1 N_{1}\left(x ; \mu_{1}, \sigma_{1}^{2}\right)+0.8 N_{2}\left(x ; \mu_{2}, \sigma_{2}^{2}\right)+0.1 N_{3}\left(x ; \mu_{3}, \sigma_{3}^{2}\right)$, where $\mu_{2}=0$, $\sigma_{2}^{2}=2, \mu_{3}=-\mu_{1}=\sqrt{3.375} \sqrt{2}, \sigma_{1}^{2}=\sigma_{3}^{2}=\frac{1}{4}$.

An overall conclusion from Table 2 is that, when the variance under the alternative is greater than the value assumed under the null hypothesis, the Kullback-Leibler test is more powerful than the Anderson-Darling test even for small sample sizes. 


\begin{tabular}{cccc|cccc}
\hline Alternative $(F)$ & $n$ & $K$ & $A^{2}$ & Alternative $(F)$ & $n$ & $K$ & $A^{2}$ \\
\hline Uniform $(-3,3)$ & 5 & 28 & 24 & Normal $(0,3)$ & 5 & 25 & 20 \\
& 30 & 65 & 59 & & 30 & 38 & 30 \\
& 50 & 84 & 80 & & 50 & 48 & 39 \\
& 90 & 98 & 97 & & 90 & 63 & 56 \\
Student $(d f=3)$ & 5 & 15 & 13 & Tukey $(\lambda=0.026)$ & 5 & 24 & 19 \\
& 30 & 24 & 20 & & 30 & 34 & 26 \\
& 50 & 30 & 25 & & 50 & 39 & 30 \\
& 90 & 43 & 38 & & 90 & 51 & 42 \\
Laplace $(b=\sqrt{1.5})$ & 5 & 22 & 18 & Mixture & 5 & 25 & 21 \\
& 30 & 31 & 23 & & 30 & 48 & 40 \\
& 50 & 37 & 28 & & 50 & 62 & 55 \\
& 90 & 47 & 37 & & 90 & 82 & 78 \\
\hline
\end{tabular}

Table 2: Empirical power comparisons, overdispersed alternative distributions

Acknowledgements. The authors wish to thank the editor and an anonymous referee for their comments and suggestions which greately improved the presentation of this paper. This work was supported by Project IN106114-3 of the Programa de Apoyo a Proyectos de Investigación e Innovación Tecnológica (DGAPAUNAM, Mexico). The first and second authors wish to also acknowledge partial support from the Sistema Nacional de Investigadores (Mexico).

\section{References}

[1] Anderson, T.W. \& Darling, D.A. (1954). A test of goodness of fit. Journal of the American Statistical Association 49, 765-769,

[2] Bahadur, R.R. (1967). Rates of convergence of estimates and test statistics. Ann. Mathem. Statist, 38, 303-324.

[3] Chui, C.K. (1971). Concerning rates of convergence for Riemann sums. Journal of Approximation Theory, 4, pp. 279-287.

[4] D’Agostino, R.B. \& Stephens, M.A. (eds.) (1986). Goodness of Fit Techniques. New York: Marcel Decker. 
[5] Grané, A. \& Tchirina, A.V. (2013). Asymptotic properties of a goodnessof-fit test based on maximum correlations, Statistics 47-1, pp. 202-215.

[6] Hill, B.M. (1968). Posterior distribution of percentiles: Bayes Theorem for sampling from a finite population. Journal of the American Statistical Association 63, 677-691.

[7] Nikitin, Y. (1995). Efficiency of Nonparametric Tests. Cambridge University Press, New York. 